\title{
Clinicopathological study of SDHB mutation-related pheochromocytoma and sympathetic paraganglioma
}

\section{Dear Editor}

Pheochromocytoma (PCC) and paraganglioma (PGL) are genetically and phenotypically heterogeneous catecholamine-producing neoplasms. They can occur sporadically or as a part of hereditary disease. Approximately $30 \%$ of PCC/PGL are believed to be caused by germline mutations (Welander et al. 2011). Of these, succinate dehydrogenase subunit $\mathrm{B}(S D H B)$ gene mutation is considered a high-risk factor for malignancy. Loss of heterozygosity at the $S D H B$ locus (1p36) was observed in all tumors with $S D H B$ mutation, and Gimenez-Roqueplo et al. (2003) strongly suggested that $S D H B$ is a tumor suppressor gene. Subsequently, loss of SDHB protein immunoreactivity in $S D H B$-mutated PCC/PGL (SDHB-PCC/PGL) was reported with $100 \%$ sensitivity and $84 \%$ specificity (van Nederveen et al. 2009). Thus, SDHB immunohistochemistry can be used to screen SDHBPCC/PGL using paraffin-embedded pathological materials. $S D H B$ mutation is the only established factor that indicates future metastasis. Therefore, it is important to analyze the histological characteristics of $S D H B-$ PCC/PGL.

It is generally accepted that it is difficult to distinguish histological differences between benign and malignant PCC/PGL. The current consensus is that a long-term follow-up is required after the surgery to screen for recurrence or metastasis in all PCC/PGL patients, regardless whether hereditary or sporadic in origin. Kimura et al. (2014) proposed a histological grading system called the Grading of Adrenal PCC and PGL (GAPP) classification for predicting metastasis. GAPP is composed of six factors: histological pattern, cellularity, presence or absence of comedo-type necrosis, vascular or capsular invasion, Ki67labeling index (\%), and elevated catecholamine type. Each factor was assigned a point and the number of points was summated. Tumor scores of $0-2,3-6$, and $7-10$ were classified into well differentiated (WD), moderately differentiated (MD), and poorly differentiated (PD) types respectively. These types correlate with tumor metastasis and patient survival. Herein, we analyzed the clinicopathological features of patients with $S D H B$ mutation using the GAPP classification and SDHB immunohistochemistry.

Fifteen Japanese patients with $S D H B$ mutation and their tumors were examined. All patients were genetically tested and confirmed to have $S D H B$ germline mutations. Patient clinical data, including age, gender, mutation sites, family history, elevated catecholamines (CA), metastasis, and present status after the initial surgical removal of the tumor, are summarized in Table 1 . The same mutations were observed in $S D H B$ exon 2 (c.137G $>\mathrm{A}$ ) and $S D H B$ exon 5 (c.470delT) in six and four patients respectively. Five of the patients (33\%) had family history. The preoperative CA phenotypes of the patients included six norepinephrine plus dopamine, six norepinephrine only, and three nonfunctioning type. Eight of the patients (53\%) had multiple distant metastases. Only two patients with multiple metastases died of the disease 37 and 12 years respectively after the initial surgery. The number of survival years from the first operation ranged from 2 to 37 years in eight patients; however, the remaining seven patients were operated upon within a past year and their prognosis could not be evaluated. Notably, four patients survived longer than 20 years with multiple metastases since the initial surgery $(20,25,26$, and 37 years respectively). Pathological data are summarized in Table 2 . Nine tumors $(60 \%)$ showed a pseudorosette pattern, which is histologically rare in ordinary PCC. Histological grading of the tumors was as follows: $1 \mathrm{WD}, 13 \mathrm{MD}$ (87\%), and $1 \mathrm{PD}$.

In previous reports, genetic testing of $S D H B$ revealed that the mutation sites were distributed throughout

Published by Bioscientifica Ltd 
Table $1 S D H B$ gene mutations and clinical data of 15 patients

Parameter
Mean age at diagno
Gender
Exon/mutation cDNA
nucleotide change

Family history of pheochromocytoma and paraganglioma

Preoperative excessive catecholamine type

Metastases

Present status

\begin{tabular}{l} 
\\
\hline $32.3 \pm 14.2(17-61)$ \\
Male \\
Female \\
Exon $2 / c .137 \mathrm{G}>\mathrm{A}$ \\
\\
Exon 5/c.470delT \\
Exon 3/c.268C $>\mathrm{T}$ \\
Exon 6/c.641A $>\mathrm{G}$ \\
Exon 7/c.649C $>\mathrm{T}$ \\
Intron2-3 \\
Intron4-5, exon5/c.424-7A $>\mathrm{C}$; \\
424-6_427del \\
Positive \\
Negative \\
Norepinephrine + dopamine \\
Norepinephrine \\
Nonfunctioning \\
Presence \\
Absence \\
Died of disease \\
Alive with disease \\
Alive without disease
\end{tabular}

\begin{tabular}{ccc}
\hline Mutation amino acid change & & $\boldsymbol{n}$ \\
\cline { 1 - 1 } & & 10 \\
R46Q & 5 \\
& 6 \\
L157 & \\
R90 & 4 \\
Q214R & 1 \\
R217C & 1 \\
& 1 \\
& 1 \\
& 1 \\
& $5(33 \%)$ \\
& 10 \\
& $6(40 \%)$ \\
& $6(40 \%)$ \\
& $3(20 \%)$ \\
& $8(53 \%)$ \\
& 7 \\
& 2 \\
& 5 \\
& 8 \\
\end{tabular}

the entire gene (Benn et al. 2006). However, exons 2 and 5 appear to be the preferential mutation sites in the present study of Japanese patients. Patients with SDHB mutations were younger, had extra-adrenal tumors more frequently, and had shorter survival times (Amar et al. 2007). However, extraordinary long-term survivors with $S D H B$ mutations and metastatic tumors have been reported. Young et al. (2002) reported a patient with SDHB mutation-associated familial malignant PGL with long survival, who was diagnosed with metastatic disease within 1 year after the initial presentation. The patient was well 30 years later, with little anti-tumor-specific therapy. Neumann et al. (2004) also reported two patients with malignant SDHBPCC/PGL that had the disease longer than 20 and 32 years respectively. The present study includes four patients with long survival up to 37 years with multiple metastases since the initial surgery. The reasons for the extraordinarily long survival with multiple metastases are important and should be clarified. Timmers et al. (2007) reported that of the 29 patients with SDHB-PHEO/PGL, the catecholamine hypersecretion profile was norepinephrine plus dopamine in 13 patients (46\%), norepinephrine only in 12 patients $(41 \%)$, dopamine only in 1 patient (3\%), and nonfunctioning in three patients $(10 \%)$. Our present findings are similar to these data. Nonfunctioning PCC/PGL might reflect immature biochemical phenotypes. Dopamine hypersecretion and plasma methoxytyramine, the O-methylated metabolite of dopamine, have been considered as the features of immaturity and a marker for malignant PCC/PGL (Eisenhofer et al. 2012).

A pseudorosette pattern is a peculiar histology that is rarely observed in ordinary PCC/PGL. A pseudorosette pattern is sometimes observed in immature tumors of other organs, including pseudo-papillary tumors of the pancreas, some pituitary tumors, and ependymomas. It is surprising that the pseudorosette pattern was observed in nine $(60 \%)$ of the 15 tumors in the present study. Thus, both catecholamine phenotype and tumor morphology revealed SDHB-PCC/PGL immaturity. Our histological analyses of SDHB-PCC/PGL revealed that most tumors (87\%) are $\mathrm{MD}$, whereas there is only one each of WD and PD tumor. Recent results from our Japanese nationwide survey of PCC/PGL have indicated that the 5-year survival rates were $100,66.8$, and $22.4 \%$ for the WD, MD, and PD types respectively (Kimura et al. 2014). Patients with MD type of SDHB-PCC/PGL may live for a long time after the initial surgery, even with multiple tumor metastases.

In conclusion, we would like to emphasize that the pseudorosette pattern in PCC/PGL and elevated norepinephrine plus dopamine or nonfunctioning are the features of SDHB mutations. Based on histopathology, the combination of SDHB-negative immunohistochemistry and the presence of pseudorosette pattern strongly suggest $S D H B-P C C / P G L$. Genomic analyses should be recommended for such cases.

Published by Bioscientifica Ltd. 
Table 2 Pathological data of pheochromocytomas and paragangliomas from 15 patients with SDHB mutation

\section{Parameter}

Tumor location

Tumor size ( $\mathrm{cm} \pm$ S.D.)

Tumor metastasis

Location of metastasis

Histologic type

GAPP score (mean \pm S.E.M.)

Histologic grade

SDHB-immunohistochemistry

Adrenal gland
Retroperitoneum
Urinary bladder
Mediastinum with pelvic cavity

Presence

Absence

Lung

Bone

Liver

Ovary and pelvic cavity

Omentum and peritoneum

Breast

Zellballen

Irregular zellballen

Pseudorosette pattern

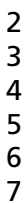

Well differentiated Moderately differentiated Poorly differentiated
Lymph node

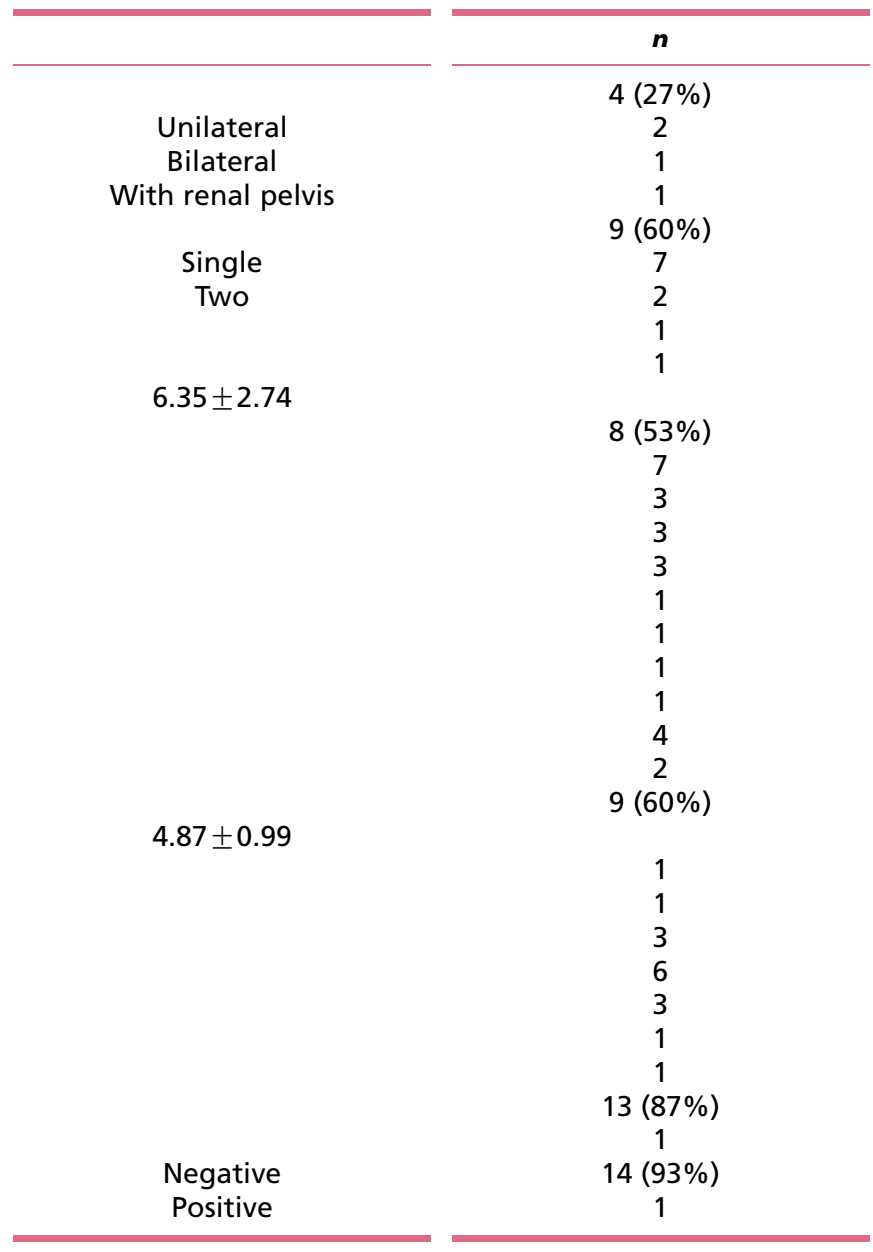

Noriko Kimura $^{1}$ Kazuhiro Takekoshi ${ }^{2}$ Akira Horii $^{3}$ Ryo Morimoto 4 Tsuneo Imai ${ }^{5}$ Yutaka Oki ${ }^{6}$ Tomohito Saito $^{7}$ Sanae Midorikawa ${ }^{8}$ Tadashi Arao ${ }^{9}$ Chiho Sugisawa $^{9}$ Masanobu Yamada ${ }^{10}$ Yuichi Otuka ${ }^{11}$ Isao Kurihara ${ }^{12}$ Kokichi Sugano $^{13}$ Minoru Nakane ${ }^{14}$ Atsushi Fukuuchi ${ }^{15}$ Takumi Kitamoto ${ }^{16}$ Jun Saito ${ }^{16}$

Tetsuo Nishikawa ${ }^{16}$ Mitsuhide Naruse ${ }^{17}$
(C) 2014 Society for Endocrinology Printed in Great Britain
${ }^{1}$ Pathology Division, Department of Clinical Research, National Hospital Organization, Hakodate Hospital, 18-16 Kawahara, Hakodate, Hokkaido, 041-8512, Japan

${ }^{2}$ Division of Sports Medicine, Faculty of Medicine, University of Tsukuba, Tsukuba, Japan ${ }^{3}$ Division of Molecular Pathology, Department of Pathology, School of Medicine, Tohoku University, Sendai, Japan ${ }^{4}$ Division of Nephrology, Endocrinology and Vascular Medicine, Department of Medicine, School of Medicine, Tohoku University, Sendai, Japan

${ }^{5}$ Department of Breast and Endocrine Surgery, Aichi Medical School of Medicine, Nagoya, Japan ${ }^{6}$ Department of Endocrinology and Metabolism, Hamamatsu Medical School, Hamamatsu, Japan ${ }^{7}$ Division of Thoracic Surgery, Department of Thoracic and Cardiovascular Surgery, Kansai Medical University, Hirakata, Japan

${ }^{8}$ Department of Diabetes, Endocrinology and Metabolism, Fukushima Medical School, Fukushima, Japan ${ }^{9}$ First Department of Internal Medicine, 
School of Medicine, University of Occupational and Environmental Health, Kitakyusyu, Japan

${ }^{10}$ Department of Medicine and Molecular Science, Graduate School of Medicine, Gunma University, Maebashi, Japan

${ }^{11}$ Department of Surgery, Nippon Telegraph and Telephone

East Corporation Medical Center, Tokyo, Japan

${ }^{12}$ Department of Internal Medicine, School of Medicine,

Keio University, Tokyo, Japan

${ }^{13}$ Genetic Counseling Clinic, National Cancer Center, Tokyo, Japan

${ }^{14}$ Department of Oncology, Musashino Red Cross Hospital, Tokyo, Japan

${ }^{15}$ Department of Breast and Endocrine Surgery, Mitsui Memorial Hospital, Tokyo, Japan

${ }^{16}$ Department of Endocrinology and Metabolism, Japan Labour Health and Welfare Organization, Yokohama Rosai Hospital, Yokohama, Japan

${ }^{17}$ Department of Endocrinology, Metabolism and Hypertension, National Hospital Organization Kyoto Medical Center, Kyoto, Japan

(Correspondence should be addressed to N Kimura; email: kimura-path@hnh.hosp.go.jp)

\section{Declaration of interest}

The authors declare that there is no conflict of interest that could be perceived as prejudicing the impartiality of the research reported.

\section{Funding}

This study was supported by grants for a Research on Measures for Intractable Diseases, Research Committee on the Diagnosis and Treatment of Pheochromocytoma from the Ministry of Health, Labour, and Welfare of Japan (M Nakane) for Advancement for Hospital Function from the Japan Health, Labour, and Welfare Organization (N Kimura), and in part from the Ministry of Education, Culture, Sports, Science, and Technology of Japan (grant number 21591168, K Takekoshi).

\section{Acknowledgements}

The authors thank K Terashima and T Moriyama for their dedication and cooperation in preparation of pathological materials.

\section{References}

Amar L, Baudin E, Burnichon N, Peyrard S, Silvera S, Bertherat J, Bertagna X, Schlumberger M, Jeunemaitre X, Gimenez-Roqueplo AP et al. 2007 Succinate dehydrogenase B gene mutations predict survival in patients with malignant pheochromocytomas or paragangliomas. Journal of Clinical Endocrinology and Metabolism 92 3822-3828. (doi:10.1210/jc. 2007-0709)

Benn DE, Gimenez-Roqueplo AP, Reilly JR, Bertherat J, Burgess J, Byth K, Croxson M, Dahia PL, Elston M, Gimm O et al. 2006 Clinical presentation and penetrance of pheochromocytoma/paraganglioma syndromes. Journal of Clinical Endocrinology and Metabolism 91 827-836. (doi:10.1210/jc.2005-1862)

Eisenhofer G, Lenders JW, Siegert G, Bornstein SR, Friberg P, Milosevic D, Mannelli M, Linehan WM, Adams K, Timmers HJ et al. 2012 Plasma methoxytyramine: a novel biomarker of metastatic pheochromocytoma and paraganglioma in relation to established risk factors of tumour size, location and SDHB mutation status. European Journal of Cancer 48 1739-1749. (doi:10.1016/j.ejca.2011.07.016)

Gimenez-Roqueplo AP, Favier J, Rustin P, Rieubland C, Crespin M, Nau V, Khau Van Kien P, Corvol P, Plouin PF, Jeunemaitre X et al. 2003 Mutations in the SDHB gene are associated with extra-adrenal and/or malignant phaeochromocytomas. Cancer Research 63 5615-5621.

Kimura N, Takayanagi R, Takizawa N, Itagaki E, Katabami E, Kakoi N, Rakugi H, Ikeda Y, Tanabe A, Nigawara T et al. 2014 Pathologic grading for predicting metastasis in phaeochromocytoma and paraganglioma. Endocrine-Related Cancer 21 405-414. (doi:10.1530/ERC-14-0494)

van Nederveen FH, Gaal J, Favier J, Korpershoek E, Oldenburg RA, de Bruyn EM, Sleddens HF, Derkx P, Rivière J, Dannenberg H et al. 2009 An immunohistochemical procedure to detect patients with paraganglioma and phaeochromocytoma with germline SDHB, SDHC, or SDHD gene mutations: a retrospective and prospective analysis. Lancet Oncology 10 764-771. (doi:10.1016/S1470-2045(09)70164-0)

Neumann HP, Pawlu C, Peczkowska M, Bausch B, McWhinney SR, Muresan M, Buchta M, Franke G, Klisch J, Bley TA et al. 2004 Distinct clinical features of paraganglioma syndromes associated with SDHB and SDHD gene mutations. Journal of the American Medical Association 292 943-951. (doi:10.1001/jama.292.8.943)

Timmers HJ, Kozupa A, Eisenhofer G, Raygada M, Adams KT, Solis D, Lenders JW \& Pacak K 2007 Clinical presentations, biochemical phenotypes, and genotype-phenotype correlations in patients with succinate dehydrogenase subunit B-associated pheochromocytomas and paragangliomas. Journal of Clinical Endocrinology and Metabolism 92 779-786. (doi:10.1210/jc.2006-2315)

Welander J, Söderkvist P \& Gimm O 2011 Genetics and clinical characteristics of hereditary pheochromocytomas and paragangliomas. Endocrine-Related Cancer 18 R253-R276. (doi:10.1530/ERC-11-0170)

Young AL, Baysal BE, Deb A \& Young WF Jr 2002 Familial malignant catecholamine-secreting paraganglioma with prolonged survival associated with mutation in the succinate dehydrogenase B gene. Journal of Clinical Endocrinology and Metabolism 87 4101-4105. (doi:10.1210/jc.2002-020312)

Received in final form 13 March 2014

Accepted 21 March 2014

Made available online as an Accepted Preprint

21 March 2014 http://erc.endocrinology-journals.org DOI: $10.1530 /$ ERC-13-0530
(C) 2014 Society for Endocrinology Printed in Great Britain
Published by Bioscientifica Ltd 